\title{
Improvement of Extinguishing way of Extensive Automatic Fire Extinguishing System for Residential Use
}

\author{
Shotaro Sadaishi ${ }^{\mathrm{a}}$, Kosuke Ando ${ }^{\mathrm{a}}$, Masahiko Hanada ${ }^{\mathrm{b}}$, Kanya Mizuguchi $^{\mathrm{b}}$, Hiromichi Hanada ${ }^{\mathrm{c}}$, \\ Seiichi Serikawa ${ }^{d}$, Yuhki Kitazono ${ }^{a, *}$ \\ a National Institute of Technology, Kitakyushu College, 5-20-1 Shii, Kokuraminami-ku, Kitakyushu-city, Fukuoka \\ 802- 0985, Japan \\ ${ }^{b}$ Hanada Setsubi, 3-25-18, Haruyoshi, Chuo, Fukuoka, Fukuoka 810-0003, Japan

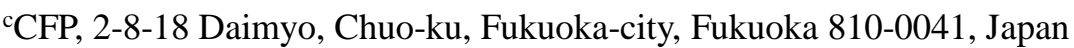 \\ ${ }^{d}$ Kyushu Institute of Technology, 1-1, Sensui-cho, Tobata, Kitakyushu, Fukuoka 804-8550, Japan \\ *Corresponding Author: kitazono@kct.ac.jp
}

\begin{abstract}
We continued to improve our previous research. It is improvement of the system which automatically detects fire and performs initial fire fighting. This system is attached to the digestive device of existing products. In this system, the nozzle is automatically directed to the fire position and automatically extinguished if it is judged to be a fire. Infrared cameras are used to judge fire, fire is determined by image processing. After extinguishing the fire, the fire information is transmitted to the smartphone via the user. In this paper, we improved this system. As a result, by devising the extinguishing method, it became possible to digest more efficiently.
\end{abstract}

Keywords: Image Processing, Fire Extinguish.

\section{Introduction}

According to the United States of National Fire Protection Association reports "Full Loss in the United States During 2017" (1), 77\% of all fire deaths occur in home fires. Fig. 1 shows the breakdown of the fire in the United States ${ }^{(1)} .38 \%$ of the fire in the United States is a structure fire, an increase of $5.0 \%$ compared to 2017 . 2630 civilians died, 14670 civilians injured, $\$ 10.7$ billion in property damage occurred in structure fires. The most effective and efficient means to prevent damage by the home fire, the initial firefighting. The advantage of the initial extinguishing in order to maximize detects the fire at an early stage, there must be a system for applying a

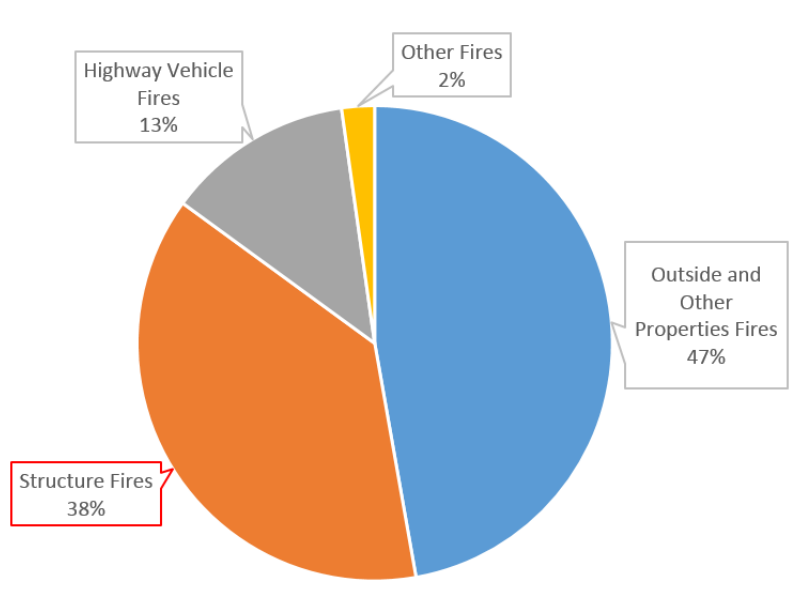

Fig. 1. Fires in the United States, 2017.

properly extinguish. Initial firefighting is that of firefighting carried out in up to two minutes before and after the fire. By applying the fire before the fire becomes large fire, it is possible to greatly reduce the damage to the fire place. Further firefighting performed by the fire also can be minimized. Therefore, it can be said to be a very efficient firefighting. Fire detectors are currently used for fire detection. Fire detector, there is a heat detector and a smoke detector $^{(2)}$. Heat detector is operating temperature has been set, if the temperature in a short period of time to jump, to detect a fire in that the cable is destroyed by heat. Therefore, it is impossible to carry out initial fire extinguishing be performed extinguishing from the detection of the fire. If the initial fire extinguishing is performed, in the case where not performed, human, leaving a large difference in size of the material damage. Based on the above, the importance of the initial fire is seen. However, the initial firefighting is not 
performed in most cases even happening fire. As the factors, if you do not notice the fire, or if that aware of the fire calm also would give priority to flee to not be able to deal with can be considered. Factors that do not notice the fire's different, but, for example, such as a fire in the fire, or without people place during sleep can be considered. In the case of such a situation, it is very difficult to perform the initial firefighting. In addition, in the home of the elderly, it is difficult to carry out the initial firefighting even if a fire occurs. In fact, in Japan is about $60 \%$ of the dead in house fire is the elderly ${ }^{(3)}$. Even in situations that allows an initial fire extinguishing elderly, it is not easy to perform properly extinguish. Because, or did not know the appropriate firefighting method, because the body may or may not move freely. Smoke detector incorporates a sample of air in the pipe, and analyzed for the presence of smoke, it is determined that a fire when it detects smoke. The heat detector and the smoke detector are both unable to detect the fire until the fire is increased. Development of universal design fire extinguisher ${ }^{(4)}$ it has also been carried out. However, it is difficult for fire-fighters while ensuring safety.

Currently, the system subjected to a fire is present a large number. For example, if the sprinkler, fire extinguishing capability is high. However, do not start a fire until the sprinkler body is heated to 70 degrees Celsius. It cannot be said that the order is carried out the initial firefighting. In addition, it carries out the fire if it is sprayed with a large amount of water. Therefore, the room together even if successful subsidence will be wetted with water. Thus, it would put a lot of damage to electronic equipment and precision equipment. Besides, the development of firefighting robots has been widely. Tokyo Fire Department of Japan is also like to have a remote control firefighting equipment georgette Fighter and intelligent ownership ${ }^{(5,6)}$. However, not for general household. The advantage of the initial extinguishing in order to maximize detects the fire at an early stage, there must be a system for applying a properly extinguish fire source.

Therefore, we aim to construct a system to perform initial fire fighting for ordinary households. As a result of the previous study ${ }^{(7)}$, in the event of a fire in the home, a fire extinguishing system was developed to allow the first fire extinguishment by remote control. In addition, improved fire extinguishing accuracy, improved fire detection system added succeeded in developing a better system. After that, we took over the research and installed a fire extinguisher on the ceiling so that we can digest not only the walls but also a wide range. However, since it is unstable whether fire can be extinguished even if you do not know the distance to the fire, we moved the hose at the time of fire extinguishment so that it could be extinguished efficiently ${ }^{(8)}$.

\section{Configuration}

The system sends images of the room from the one infrared camera to computer. The camera moves at a constant cycle and acquires surrounding images. Then, each image processing is performed. When a fire is detected, the user can see the real-time video in the room from the video camera. Based on the image, check whether a fire is occurring. If a fire is confirmed, extinguish the fire. When extinguishing fire, control each motor by Arduino. The motor uses one for moving the hose of the fire extinguisher up and down and the other two for moving it to the left and right. By doing this, it manipulates the direction and trigger of the fire extinguisher's hose. When extinguishing a fire, fire extinguishing agent from a fire extinguisher while swaying to the left and right from the front of the fire source like sweep with a broom.

One is a fire detection unit and the other is a fire extinguishing unit. The configuration of the system is shown in Figs. 2 and 3. The fire detector consists of one

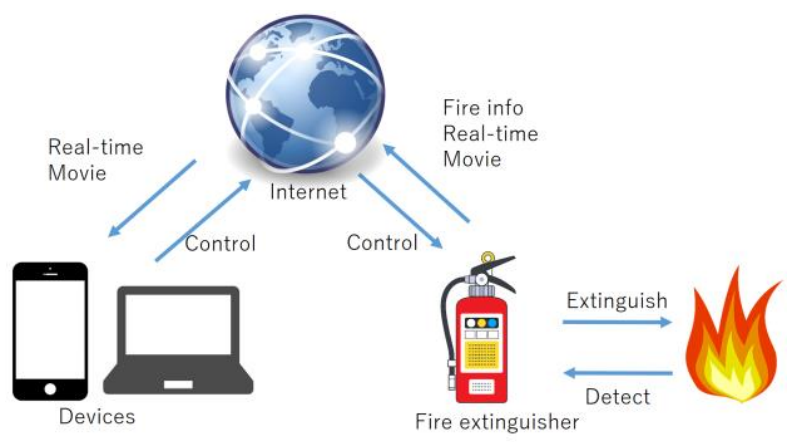

Fig. 2. Operation overview of relation.

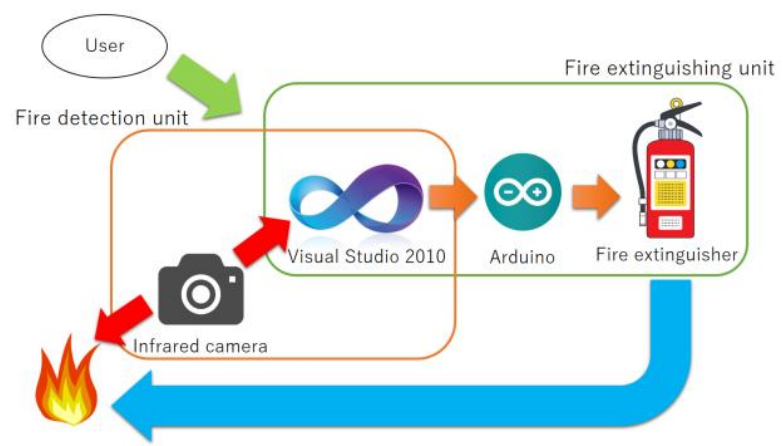

Fig. 3. Structure of the system. 
infrared cameras as shown in Fig. 4 and Visual Studio 2010. The infrared camera is used to detect a fire. Since the flame is emitting infrared light, the infrared camera can see the shape of the infrared so you can know the size of the flame. Image processing is applied to the infrared image obtained by the infrared camera and the size thereof is discriminated

to detect the presence or absence of the flame. Image processing uses OpenCV. The language used is $\mathrm{C}++$. The method of fire detection is shown below. First, the image from the infrared camera is imported into Visual Studio 2010. The image of the infrared camera shows the infrared ray emitted from the flame. The image is processed by OpenCV. The processing flow is shown below. The image of the infrared camera is stored in each pixel 0 convert to a grayscale with a value of $0 \sim 255$. The converted image is compared with a threshold set in advance in the program. By comparing, infrared other than flame is removed. In other words, pixels below the threshold value are represented as black, pixels above the threshold value as white. This is called binarization. Binarization makes it easier to distinguish the shape of the flame. By the processing so far, only the flame shape is extracted from the image from the infrared camera. This makes it possible to judge whether or not a fire has occurred. In this system, the area of the white part of the binarized image is calculated. The contour of the white part is detected with OpenCV, and the area inside it is calculated. At this time, even when a plurality of infrared rays are detected, only the infrared ray having the largest area is calculated. We will move on to extinguishment only when the area over the pre-determined threshold is observed. Therefore, even if there is something generating a strong infrared ray such as a stove or a gas stove, it is possible to set a threshold in advance so that malfunction can be prevented. When a fire is detected, a notification is sent to the mail address registered in advance

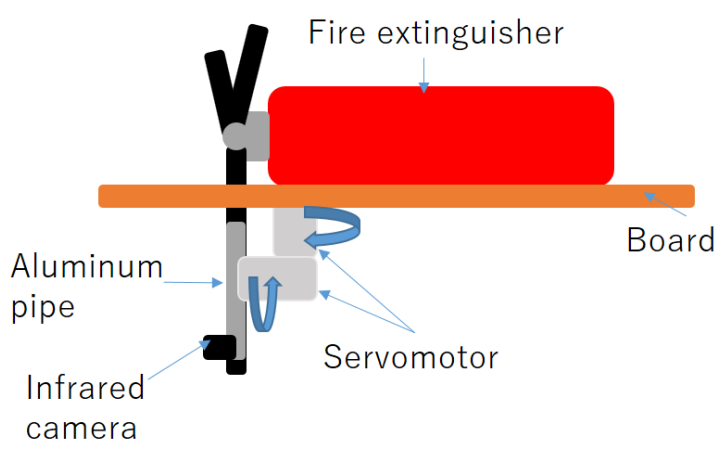

Fig. 4. Illustration of structure.
Table 1. Used equipment.

\begin{tabular}{|c|c|c|}
\hline Equipment & Maker & Model Number \\
\hline Arduino & Arduino & MEGA ADK \\
\hline Camera & ELECOM & UCAM-DLE300T \\
\hline Servo Motor & GWS & GWS777FCG/6BB \\
\hline IR Filter & FUJIFILM & IR 96 \\
\hline
\end{tabular}

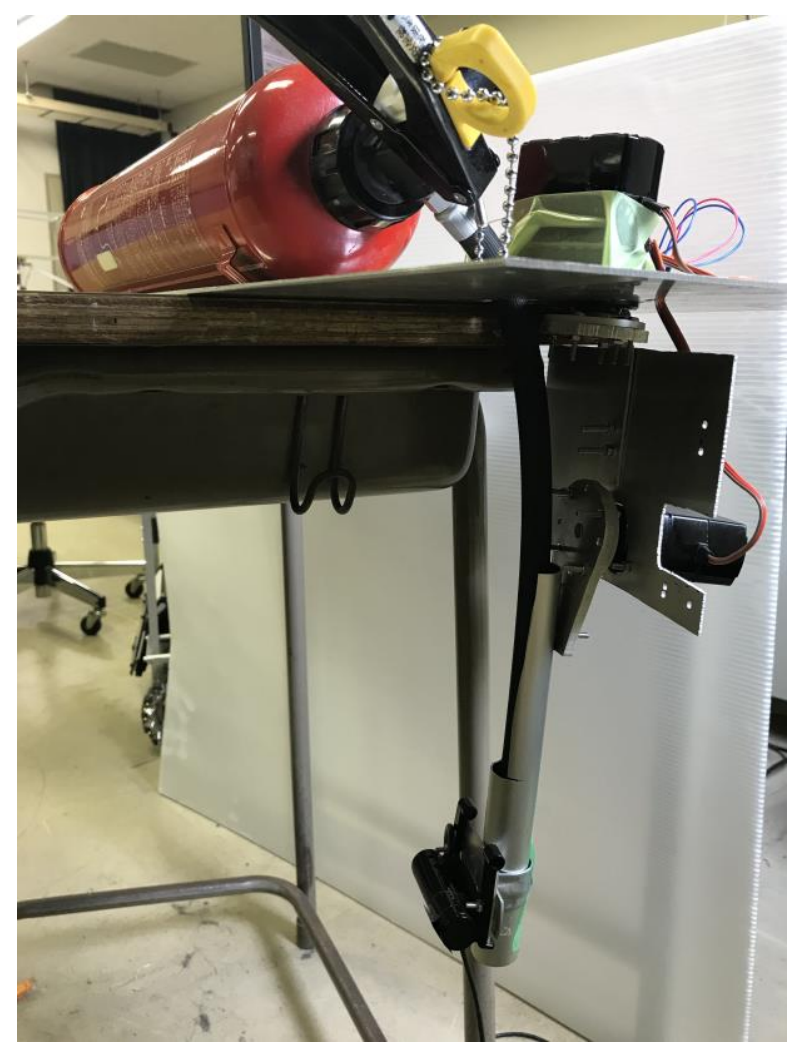

Fig. 5. Actual structure.

in the system. The user accesses the website described in the e-mail. This website is a site for remotely controlling the fire extinguishing system and it is possible to confirm with real time the fact that a fire is occurring on this website before extinguishing fire. Immediately after the references are made. When extinguishing a fire, firefighting can be done using a command to move the hose of a fire extinguisher on the website up and down or left and right or a command to fire extinguishing agent.

\section{Experiments and Results}

Experiments were conducted to ascertain whether the improved parts are functioning properly. In this system, we used equipment as shown in Table 1.

\subsection{Operation Experiment of System}

In this study, we improve fire extinguishing method from previous research and extinguish efficiently by 


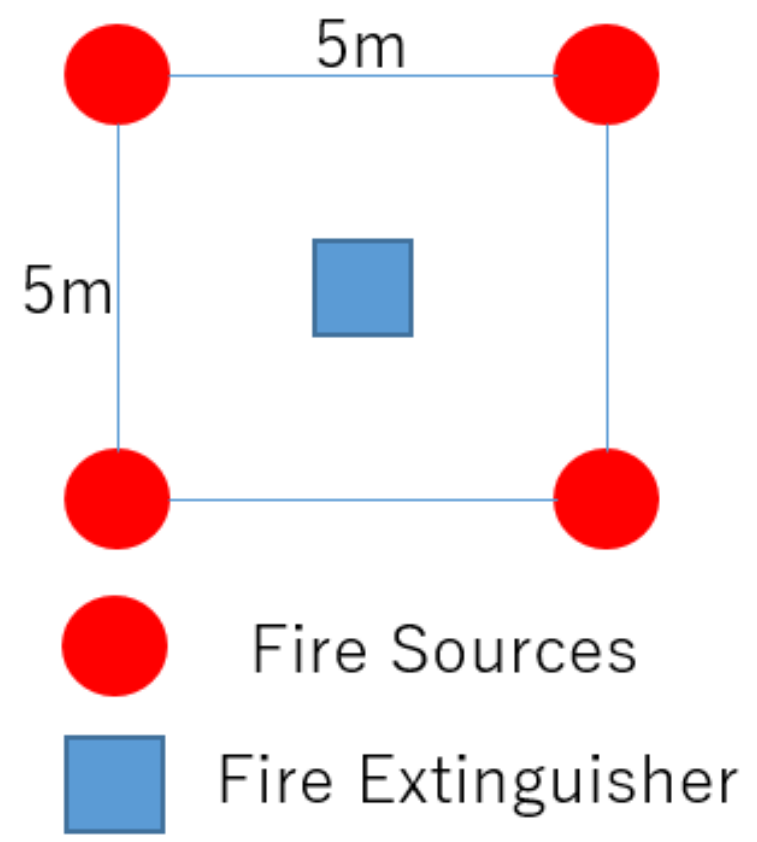

Fig. 6. Experiment schematic

moving hose while spraying fire extinguishing agent. Move the camera with the motor to find the fire sources and extinguish it, but at that time you cannot extinguish efficiently unless you make predetermined movements. Therefore, when detecting a fire, I experimented whether the motor works correctly. The experimental schematic is shown in Fig. 6. A fire extinguisher was set at a point $1 \mathrm{~m}$ above the ground and the fire sources were set as shown in the figure. In this state, an experiment was conducted as to whether the motor is performing specified motion. As a result, 20 experiments were performed for each fire sources, but it worked normally. From this, it is understood that the fire extinguisher is operating normally.

\section{Conclusions}

In this research, we successfully extinguished more effectively by improving fire extinguishers developed so far. This makes it possible to efficiently perform the initial fire extinguishment. But there are still some points to improve. In this research we use a fire extinguisher and a powder fire extinguisher, but in the case of powder, we do not know whether the fire has disappeared after the powder adheres to the camera after spraying on the source of fire, so we want to improve that point. We want to improve the disadvantages one by one and we want to make better ones.

\section{References}

(1) Ben Evarts, "Full Loss in the United States During 2017”, October 2018

(2) http://www.kaho.or.jp/user/eq/eq02/p01.html

(3) Ministry of Internal Affairs and Communications Fire and Disaster Management Agency, the fire in 2015 (January to December) Overview (approximate), July 2016

(4) Japan fire extinguisher Industry Association, "the development of universal design fire extinguisher", Monthly fescue, No.397 pp.30-35, 10 May 2014

(5) Yoshinori Takei, Yasunori Takayama, Tetsuro Tashiro, Minato Hidehito, Sachiyo Iwasaki, Hiroshi Kasahara, "the development of odor sensor system for fire detection and study of the robot-mounted", Electronics, Information and Communication Engineers Technical report .MBE, ME and Bio Cybernetics, 597 No., No.MBE2002-107, pp.45-48, May 2003.

(6) Tokyo disaster prevention equipment Co., Ltd., automatic firefighting robot http://www.tokyo-bosai-setsubi.co.jp/tbs/seihin/ia_tips. html

(7) Kosuke Ando, Masahiko Hanada, Hiromichi Hanada, Kanya Mizuguchi, Seiichi Serikawa, Yuhki Kitazono, "Full Automatic Wide Range Fire Extinguish System for Residential Use", Proceedings of the 5th IIAE International Conference on Intelligent Systems and Image Processing 2017, 2017.

(8) Yokohama municipal fire department, How to use fire extinguisher

http://www.city.yokohama.lg.jp/shobo/seikatsu/shokish ouka/shoukaki.html 